\section{Angst in der Kunst. Ikonographie einer Grundemotion}

Katharina Domschke. Angst in der Kunst. Ikonographie einer Grundemotion. Stuttgart: Kohlhammer 2019. 200 Seiten, 49,00 Euro, ISBN 978-317-035-150-9

Dass Ärzte über den Tellerrand ihres Handwerkes bzw. ihrer medizinisch-therapeutischen Wissenschaft schauen und über die Grenzen der Disziplin hinaus geistig-kreativ tätig werden, zeichnet - charmanten Stereotypen entsprechend - diese unsere Berufsgruppe aus. Auch die Autorin, anerkannte Expertin auf dem Gebiet der Angststörungen und Lehrstuhlinhaberin in Freiburg, hat mit dem vorliegenden Buch „Angst in der Kunst“" einen entsprechenden transdisziplinären Vorstoß unternommen. Unter dem Titel, der den universellen Anspruch einer Ikonographie und damit eine systematische Arbeit impliziert, findet sich zum einen ein laienverständlicher Überblick über den aktuellen Forschungsstand zum Thema Angststörungen und deren Behandlung. Kunst wird hier als eine Facette von Prävention bzw. als Angst-Heilmittel eingeführt. Es folgt eine erklärtermaßen subjektive Auswahl von Kunstwerken überwiegend aus dem späten 20. und frühen 21. Jahrhundert, die jeweils von assoziativen Texten mit kunsthistorischen, therapeutischen, soziologischen und philosophierenden Inhalten begleitet werden. In diesen Texten findet man vieles, was der
Überlegung und Reflexion wert ist. Leser, die assoziativ Anregungen suchen, um über das Thema Angst zu reflektieren, werden reichhaltig fündig. Leser, die mehr suchen, zumal eine dem Titel entsprechende fundierte Arbeit, werden jedoch enttäuscht. Dass auch der Beitrag der Kunsthistorikerin Martina Padberg assoziativ bleibt und, trotz Nennung zahlreicher Künstler diverser Epochen an dem, was Kunstwissenschaften idealerweise ausmacht, vorbeigeht (u. a. methodischem Vorgehen und dem Bewusstsein dessen, dass Kunst aus Kunst entsteht) erstaunt. Seit den apotropäischen „Kunst“-Werken der Steinzeit versuchten Menschen einen kreativen Umgang mit der Grundemotion Angst. Religiöse Kunst, von dem notorischen Versuch etwa der alten Ägypter, Todesangst durch bildnerische Darstellung des Lebens in gelebte Unendlichkeit zu transformieren, bis zum unendlichen Spektrum christlicher Kunst, in dem Angst ein zentrales Motiv ist (z. B. Christus am Ölberg, Jüngstes Gericht, gemarterte Heilige) und dadurch (versuchsweise) gebannt wird: Aus diesen Motiven ergäbe sich eine Grundlage, auf der dann die diversen postmodernen Werke zum Thema hätten gespiegelt werden können. Im Detail ließe sich sehr vieles anmerken. Etwa: Warum soll die „Sorgende Frau“ von Ernst Barlach von 1910 antizipierend auf das Schicksal des (später „entarteten“) Künstlers im dritten Reich verweisen? Hatten einfache Leute - „Die Weber“ von Gerhard Hauptmann erschien 1892 - in der Kaiserzeit kei- nen Grund für Angst und Sorge? Dass u. a. „Der Schrei“ von Edvard Munch nicht aufgenommen wurde, wird damit begründet, dass die Angst darin „eher implizit dargestellt" sei. Wenn dafür ein Bild von Karl Schmidt-Rottluff erscheint, das ein Stillleben mit (pseudo) afrikanischer Maske zeigt und den Titel „Panischer Schrecken“ (angesichts der ruhig daneben liegenden Pfeife und anderer Utensilien) bestenfalls mit humoristischer Note trägt, dann überzeugt die im- und explizit Argumentation nicht. Insgesamt mag man bedauern, dass die Möglichkeit vertan wurde, ein angstfrei sehr groß gewähltes Thema (zumindest) annäherungsweise systematisch aus medizinischer und kunsthistorischer Perspektive heraus anzugehen. Dass Kriegsbilder, von Francisco di Goya über Otto Dix bis Bernhard Heisig, von der Autorin komplett ausgespart wurden, Werke, deren existenzielle Angst-Vehemenz Betrachter in ihren Bann zieht und die schon deshalb in einer Ikonografie der Angst unverzichtbar wären, zeigt die Schmerz- bzw. Angstgrenze der Autorin auf, die ihrem Thema gegenüber letztlich auf ästhetischer, intellektueller und leitlinienkonformer ärztlicher Distanz bleibt. Abgesehen davon darf man sich über den sehr persönlichen, souveränen Mut zur Lücke zeigenden Zugang der Autorin freuen, die in dem Buch vieles bringt und entsprechend manchem (Leser) etwas bringen wird.

Andreas Hillert, Prien am Chiemsee 